\title{
Power Control with Random Delays: Robust Feedback Averaging
}

\author{
Andrew Ward, Zhengyuan Zhou, Panayotis Mertikopoulos, and Nicholas Bambos
}

\begin{abstract}
Distributed power control schemes in wireless networks have been well-examined, but standard methods rarely consider the effect of potentially random delays, which occur in almost every real-world network. We present Robust Feedback Averaging, a novel power control algorithm that is capable of operating in delay-ridden and noisy environments. We prove optimal convergence of this algorithm in the presence of random, time-varying delays, and present numerical simulations that indicate that Robust Feedback Averaging outperforms the ubiquitous Foschini-Miljanic algorithm in several regimes.
\end{abstract}

\section{INTRODUCTION}

Proper management of wireless communication networks relies on efficient and robust power control strategies. Such strategies may be broadly categorized into either centralized or distributed paradigms. While centralized methods may allow for more globally optimal theoretical results, this is counterbalanced by an increased communication requirement for implementation, and a reduction in system robustness due to the presence of a single total failure point. In contrast, divesting the control into a distributed scheme alleviates these concerns and opens up the study of both cooperative and competitive optimal decentralized control strategies [1], [2]. Such strategies have been well-examined, and solutions for the fundamental problems have been established.

One foremost power control method in such a distributed wireless network was developed by Foschini and Miljanic (FM) [3]. Since its development, the FM algorithm has proved applicable and stable in far more general environments than originally considered [4]. As a clean and polished control scheme which possesses several strong and useful convergence properties, the FM algorithm has heavily inspired subsequent research in the field [5], [6], [7]. Extensive studies have examined the power control problem from game-theoretic and utility based perspectives, allowing the consideration of cooperative and competitive network participants [8], [9], [10], [11]. These control methods generally focus on "memoryless" implementations, which only consider the current power signal vector when deciding the optimal power for a subsequent iteration. This restriction results in well-known stability issues.

In a 2017 study which addresses these stability issues, Zhou et al. presented a power control algorithm known as dual averaging (DA) [12]. This algorithm uses historical

A. Ward, Z. Zhou, and N. Bambos are with the Department of Electrical Engineering, Stanford University, Stanford, CA, 94305, USA. \{atward, zyzhou, bambos\}estanford.edu P. Mertikopoulos is with the French National Center for Scientific Research (CNRS) and with Univ. Grenoble Alpes, CNRS, Inria, Grenoble INP, LIG, F-38000, Grenoble, France. A. Ward is funded by the Department of Defense through an NDSEG fellowship information in a way that prevents the current transmitted power from being dominated by any particular previously received signal. The algorithm strictly enforces fully distributed control and eliminates extraneous communication requirements. Furthermore, the total historical information is not stored explicitly, but is carried forward implicitly and efficiently in rolling summaries to reduce memory requirements.

A further concern beyond memory requirements is the delays inherent in any physically realizable system. These delays can result from either signal propagation time or from the computational requirements to implement such power control algorithms in any real-world device. Previous literature has studied the effect of relatively simple constant and time-varying delays on the FM algorithm and adaptions thereof [13], [14], [15], [16]. However, modern mobile and mesh wireless networks may consist of a wide range of distinct devices, each attempting to send different types of information. This may lead to a spread of delays culminating in either no power signals in a time slot, or multiple signals being delayed to the same subsequent time slot. Furthermore, it could also lead to power signals arriving out of order. Since the presence of delay is so ubiquitous in real-world systems, any new power control algorithm should be shown to handle the concerns that delayed information introduces. Several previous studies have presented neural-network techniques to combat time-varying delay [17], [18], but these can be computationally intensive and require parameter tuning.

Our goal is to present a new power control algorithm that maintains the desirable stability and efficiency of the DA algorithm [12], and is capable of handling arbitrary delays. We must first address the situation in which multiple power iterates are delayed such that they arrive within a single time step. In this case, the control algorithm must either choose from or combine all available data without prior knowledge of the order in which the iterates were generated. We must also allow for the situation in which power iterates are received out of order. These considerations allow our new algorithm to address a wide range of both deterministic and random delays, a highly desirable capability which greatly increases the robustness of the system.

Delays have been studied in wireless networks in the context of the FM algorithm [13], [14], [15], [16], though none have addressed the case in which multiple SINR measurements arrive simultaneously or the case where SINR measurements arrive out of order. So far, properties of the DA algorithm in a network with delays have not been studied. 


\section{A. Our Contributions}

Our contributions are threefold: we first present Robust Feedback Averaging (RFA), a novel algorithm which utilizes the core idea of the dual averaging distributed power control algorithm. This new algorithm expands the DA algorithm's capabilities, and allows it to address a wide range of delay types. RFA is a fully asynchronous control algorithm, and this asynchronicity is central in RFA's ability to handle arbitrary delays. This property relaxes the need for update synchronization, and even allows different links to perform power updates at different rates. Furthermore, the RFA algorithm maintains both the constant memory property and the baseline power convergence guarantee of the original DA algorithm in this more general environment.

Second, we prove that the RFA algorithm maintains the original performance guarantees: if the channel is feasible, the RFA algorithm converges to the optimal power vector. In addition, we prove a new guarantee for the RFA algorithm: even subject to random delays, the algorithm converges almost surely to the optimal power vector. These guarantees are not matched by the FM algorithm, or by parallel adaptations of such. While the FM algorithm has been shown to converge under certain time-varying delays, it may fail to converge when said delays are arbitrarily random. Additionally, the FM algorithm as it stands cannot address the situation of multiple iterates within a time slot, and even if augmented in a manner similar to the RFA algorithm, does not maintain its convergence guarantee.

Thirdly, we demonstrate the robustness of the RFA algorithm in the presence of both random channels and delays via a series of numerical simulations. These simulations demonstrate that the RFA algorithm achieves convergence in a variety of scenarios, both ones that we prove will converge and ones where the RFA algorithm is not proven to converge. We also show that our algorithm empirically outperforms a corresponding augmentation of the FM algorithm.

\section{BACKGRound, Model, Motivation}

\section{A. Power Control in Wireless Networks}

We consider a typical wireless network model consisting of $N$ communication links, each comprised of a transmitter and an intended receiver. The transmitted power vector is denoted by $\mathbf{p}=\left(p_{1}, \ldots, p_{N}\right)$ where $p_{i}$ is the power used by the transmitter of link $i$. For this paper, we consider only non-negative real transmission powers. We also enforce a maximum power $p_{i}^{\max }$ for each link, which is a standard practical constraint [12] [19]. The feasible support set for the power vector, then, is $\mathcal{P}=\prod_{i=1}^{N}\left[0, p_{i}^{\max }\right]$.

The most common service quality measure used in power control systems is signal to interference and noise ratio (SINR). Given a power vector $\mathbf{p}$, link $i$ 's $\operatorname{SINR} r_{i}(\mathbf{p})$ is given by the following ratio:

$$
r_{i}(\mathbf{p})=\frac{G_{i i} p_{i}}{\sum_{j \neq i} G_{i j} p_{j}+\eta_{i}}
$$

where $\eta_{i}$ is the thermal noise associated with the receiver of link $i$ and $G_{i j} \geq 0$ is the power gain between transmitter $j$ and receiver $i$; for $i \neq j, G_{i j}$ represents the interference receiver $i$ experiences due to transmitter $j$ per unit transmission power used. Here, we represent all the power gains $G_{i j}$ with the gain matrix $\mathbf{G}$ and all the thermal noises with the vector $\eta$.

Additionally, each link $i$ has a threshold $r_{i}^{*}>0$, which denotes the minimum SINR necessary to achieve acceptable service quality for link $i$. The standard power control problem [20], [21] is to find a power vector $\mathbf{p}$ such that the quality of service constraints hold:

$$
r_{i}(\mathbf{p}) \geq r_{i}^{*}, \forall i
$$

where inequality is component-wise. Finding a solution to this problem assumes that such a power vector exists; to characterize the existence of such power vectors, we shall define the notion of channel feasibility:

Definition 1. A channel, given by $\mathbf{G}, \eta$ is said to be feasible with respect to a target SINR vector $\mathbf{r}^{*}=\left(r_{1}^{*}, \ldots, r_{N}^{*}\right)$ if there exists a p satisfying (2). The channel is otherwise said to be infeasible.

We can easily check for channel feasibility by constructing the re-weighted noise vector $\gamma$ and the re-weighted gain matrix $\mathbf{W}$ :

$$
\gamma_{i}=r_{i}^{*} \frac{\eta_{i}}{G_{i j}} \quad W_{i j}= \begin{cases}0 & , i=j \\ r_{i}^{*} \frac{G_{i i}}{G_{i j}} & , i \neq j\end{cases}
$$

Based on [3], we have the following definition, which gives us a convenient way to check channel feasibility:

Definition 2. A channel $\{\mathbf{G}, \eta\}$ is feasible with respect to a target SINR vector $\mathbf{r}^{*}$ if and only if the largest eigenvalue $\lambda_{\max }(\mathbf{W})<1$.

We also define the optimal power vector $\mathbf{p}^{*} \in \mathcal{P}$ to be the smallest component-wise $\mathbf{p}$ that satisfies (2) and lies in the feasible support set.

\section{B. Power Control Algorithms}

The standard algorithm used in distributed power control systems is the Foschini Miljanic (FM) algorithm [3]. The FM power update rule, modified slightly to accommodate the maximum power bounds, is

$$
p_{i}^{t+1}=\min \left(p_{i}^{t} \frac{r_{i}^{*}}{r_{i}\left(\mathbf{p}^{t}\right)}, p_{i}^{\max }\right)
$$

A novel alternative update to the FM algorithm is the dual averaging (DA) power control algorithm [12], which was inspired by the dual averaging/mirror descent optimization algorithm [22], [23], [24] . This algorithm performs updates on a dual power vector, $\mathbf{y}$, and then transforms these dual power vectors into the transmitted power vector $\mathbf{p}$ by finding the closest $\mathbf{p}$ to $\mathbf{y}$ that satisfies the constraints given in (2) and is feasible. The dual averaging update equation is:

$$
y_{i}^{t+1}=y_{i}^{t}-\frac{1}{t+1}\left(G_{i i} p_{i}^{t}-r_{i}^{*}\left(\sum_{j \neq i} G_{i j} p_{j}^{t}+\eta_{i}\right)\right),
$$

and these dual power vectors are simply clipped to ensure feasibility, i.e. $p_{i}^{t}=\max \left(0, \min \left(y_{i}^{t}, p_{i}^{\max }\right)\right)$. 


\section{Delay in Wireless Networks}

While both the FM algorithm and the DA algorithm enjoy optimal convergence properties in a delay-free network, realworld networks include multiple sources of latency. As they stand, neither the FM update presented in Equation 4 nor the DA update shown in Equation 5 is able to accommodate delays; both of these update equations assume the SINR $r_{i}\left(\mathbf{p}^{t-1}\right)$ from the previous iteration, measured at receiver $i$, is immediately available to the transmitter at time step $t$. Furthermore, the power updates are assumed to be synchronized across all links in the system.

We aim to explore the properties of the dual averaging algorithm in the presence of delayed SINR measurements. However, before we present our Robust Feedback Averaging algorithm, we must formally introduce the notion of delay in our model.

We now consider a channel in which, for each time step and for each link, the SINR signal is delayed by $D_{i}^{t} \in \mathbb{Z}^{+}$ units. Notice that these delay variables are distinct for every transmitter $i$ and every time step $t$. Therefore, if transmitter $i$ sends a power vector $\left(p_{i}^{t^{\prime}}\right)$ at time $t^{\prime}$, that transmitter will receive the SINR measurement $r_{i}\left(\mathbf{p}^{t^{\prime}}\right)$ from receiver $i$ at time $t^{\prime}+D_{i}^{t^{\prime}}$.

This is a more comprehensive model of delay than those previously considered in the context of the FM algorithm [13], [14], [15], [16]. In these works, the delay was either constant or varying with respect to time, but not also varying with respect to each receiver. These conveniently avoid the aforementioned issues we have highlighted in this paper: simultaneous SINRs and out-of-order SINRs.

The notation presented here allows for a general model for delay, and can account for the both of these cases. Additionally, by making $D_{i}^{t}$ a random variable, we can explore the effect of random delays on our algorithm.

\section{Robust Feedback Averaging With Delayed AND ASYNCHRONOUS UPDATES}

We now discuss the Robust Feedback Averaging algorithm. In the traditional formulation of both the FM algorithm and the DA algorithm, the SINR $r_{i}\left(\mathbf{p}^{t}\right)$, is assumed to be immediately available to the transmitter at every time step, and updates are assumed to be synchronized across all links in the system. By adjusting the DA algorithm for delayed, asynchronous updates, we can relax these two assumptions.

The RFA algorithm, shown in Algorithm 1, is able to accommodate these delayed SINR measurements. Since the delays $D_{i}^{t}$ can all be different, it is possible for a link to receive no signals at all on a given time step. Conversely, it is also possible for a link to receive multiple SINR measurements in a given time step. When no new SINR signals are received by transmitter $i$, the value of $y_{i}$ (and the transmitted power $p_{i}$ ) do not change. When multiple SINR measurements are received, they are simply averaged.

To perform these two actions, we calculate $n$, which represents the number of SINR measurements received by a transmitter in a given time step. If the transmitter has received any measurements, then an average is taken and the dual power vector is updated as shown in Line 7 of Algorithm 1. Noticeably, the algorithm doesn't know which SINRs were received, nor if the SINRs arrive out of order. Because of the running average implicit in the dual power vectors $y_{i}$, the algorithm can dynamically adapt to whatever SINR measurements it receives, regardless of the order. This implicit storing of all historical power and update information is a necessary practical detail in the context of memory-constrained wireless transmitters.

In addition to the minimal memory requirements, the RFA algorithm as presented satisfies a fundamental requirement: it converges to the optimal power vector $\mathbf{p}^{*}$ in the presence of random delays.

Theorem 1. Let $\mathbf{p}^{t}$ be the iterates generated by Algorithm 1 . If the random delays are all bounded: $D_{i}^{t}<\infty, \forall i, t$, then $\mathbf{p}^{t} \rightarrow \mathbf{p}^{*}$, a.s..

The proof is omitted due to space limitation. The property of almost sure convergence to the optimal vector makes the RFA algorithm a powerful tool in delay-ridden networks. Furthermore, the only condition required of the proof is that the delays be finite. In real-world systems, this is always true, so this is an extremely weak assumption. And, in the presence of no delay, RFA reduces to the dual averaging algorithm and enjoys almost sure convergence in both deterministic and stochastic time-varying channels.

\section{Simulation Results}

To validate the convergence and performance guarantees of Robust Feedback Averaging, we provide several numerical experiments that illustrate the algorithm's various properties. We consider two sets of experiments in this section: first, we introduce two different forms of delay into a deterministic, time invariant channel; second, we examine the effect of adding delays in a more complex stochastic and time-varying channel.

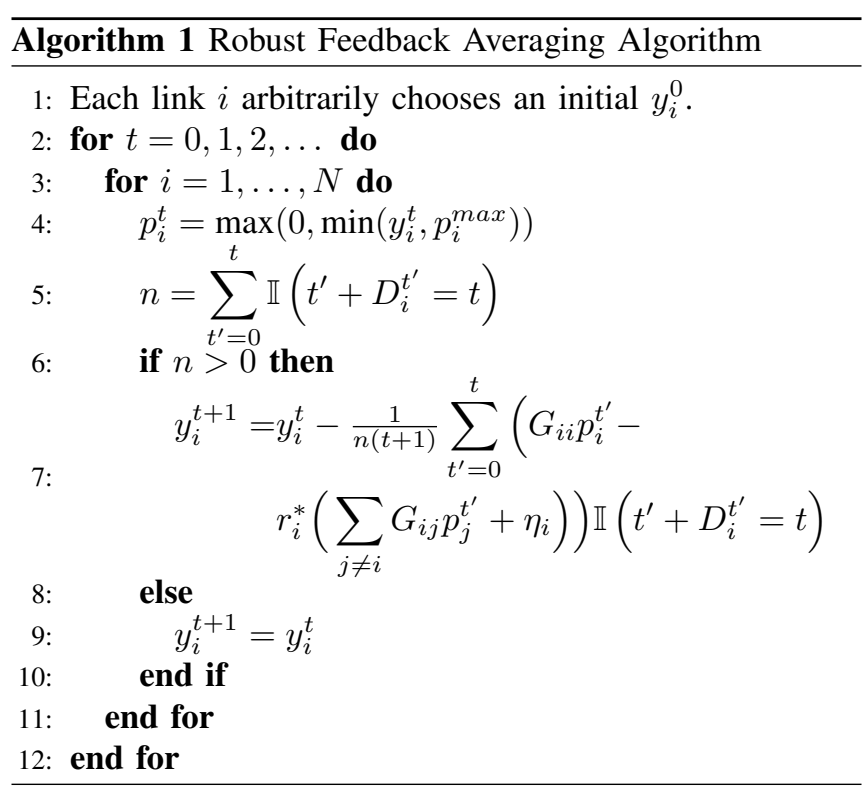




\section{A. Foschini-Miljanic Algorithm with Asynchronous Delay}

As an algorithmic comparison, we also alter the FoschiniMiljanic algorithm to account for transmitter-distinct, timevarying delays. We further modify the FM update presented in [13] to allow the FM algorithm to handle delays which are specific for each link. Our modification is similar to the modification in Algorithm 1: all of the SINR values returned in a given time step are averaged, and the average value is used for the FM update. The modified FM update rule is:

$$
p_{i}^{t+1}=\frac{1}{n} \sum_{t^{\prime}=0}^{t}\left(p_{i}^{t^{\prime}} \frac{r_{i}^{*}}{r_{i}\left(\mathbf{p}^{t^{\prime}}\right)}\right) \mathbb{I}\left(t^{\prime}+D_{i}^{t^{\prime}}=t\right)
$$

where $n=\sum_{t^{\prime}=0}^{t} \mathbb{I}\left(t^{\prime}+D_{i}^{t^{\prime}}=t\right)$ as before.

As expected, our modified FM algorithm reduces to the original FM algorithm when $D_{i}^{t}=0 \quad \forall i, t$. We compare the performance of this modified FM algorithm and our RFA algorithm in the following experiments.

\section{B. Deterministic and Time-Invariant Channel}

We begin with a deterministic, time-invariant channel with 4 links. The channel properties $\mathbf{G}, \eta$ and optimal power vector $\mathbf{p}^{*}$ are

$$
\mathbf{G}=\left[\begin{array}{llll}
3 & 1 & 2 & 1 \\
1 & 3 & 1 & 2 \\
2 & 1 & 3 & 1 \\
1 & 2 & 1 & 3
\end{array}\right] \eta=\left[\begin{array}{l}
0.1 \\
0.2 \\
0.3 \\
0.1
\end{array}\right] \mathbf{p}^{*}=\left[\begin{array}{l}
0.079167 \\
0.089583 \\
0.104167 \\
0.077083
\end{array}\right]
$$

For these simulations, we select the target $\operatorname{SINR} r_{i}^{*}=0.5$ and the maximum power $p_{i}^{\max }=1.0$ for all links in the channel. We chose these parameters to present a relatively challenging environment for the algorithms; as the $\mathbf{G}$ matrix indicates, each link experiences a lot of interference from every other link in the system. Due to this challenging environment, we only require the links to achieve a SINR of 0.5 . The maximum power $p_{i}^{\max }=1.0$ was chosen by considering the optimal power vector $\mathbf{p}^{*}$; any algorithm which finds the optimal transmitter powers will not be affected by this upper bound in the long run.

We can calculate the re-weighted gain matrix and reweighted noise vector $\mathbf{W}, \gamma$, and verify that $\lambda_{\max }(\mathbf{W})=$ $0.67<1$. Therefore, based on Definition 2, the channel is stable. This means that, for both the FM algorithm and the RFA algorithm, the transmitted link powers will converge to the optimal values in the absence of delay.

We will examine algorithm performance on this channel in several different delay regimes. For the simulations shown, all powers $p_{i}^{0}(\mathrm{FM})$ and dual powers $y_{i}^{0}$ (RFA) were initialized to zero.

Experiment 1: We first examine the algorithmic performance under the regime of constant delay. However, we make the delay distinct for each channel. For this case, we set $\mathbf{D}=\left[\begin{array}{llll}D_{1} & D_{2} & D_{3} & D_{4}\end{array}\right]^{T}=\left[\begin{array}{llll}25 & 50 & 75 & 100\end{array}\right]^{T} \quad \forall t$. This is a reasonable experiment, as in a practical setting, each transmitter-receiver pair could have different computational capabilities or could be separated by a greater physical distance, causing the delays for each link to be distinct.

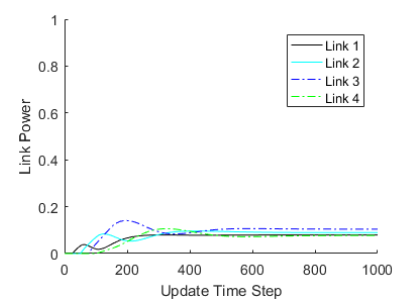

(a) Robust Feedback Averaging

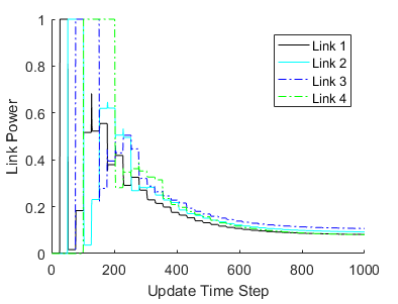

(b) Foschini-Miljanic
Fig. 1: Results of Experiment 1: power marginal convergence behavior of the RFA algorithm (a) and the FM algorithm (b) with a fixed, constant delay for each channel: $\mathbf{D}=\left[\begin{array}{llll}25 & 50 & 75 & 100\end{array}\right]^{T} \quad \forall t$ for channels 1-4, respectively. Both algorithms converge to the optimal power vector, and RFA uses less overall power.

Even this simple scenario is not covered by the works which studied the FM algorithm in the presence of delay [13], [14], [15], [16], so the FM algorithm is not guaranteed to converge. However, as we proved in Theorem 1, RFA will eventually return the optimal power vector $\mathbf{p}^{*}$.

Figure 1 shows the power for each link with this constant delay. Specifically, Figure 1(a) shows the transmitted power for each link plotted against time when using the RFA algorithm, and Figure 1(b) does so using the modified FM algorithm. As expected, neither algorithm updates until the $25^{\text {th }}$ time step, when the first SINR is received. At $t=25$, only Link 1 updates, as it is the only one to receive a SINR measurement; 25 time steps later, Link 2 begins updating, then Link 3, and finally Link 4.

The FM algorithm exhibits interesting, step-wise behavior in Figure 1(b). This is due to the fact that the FM algorithm only depends on the most recent SINR measurement to set its next transmission power. Because the transmission powers at time $t=[0,25)$ were all the same, the SINRs received during $t=[25,50)$ were all the same, so the transmission powers in that interval remained constant.

Regardless of the stepwise behavior, the power appears to converge to the optimal values both while using the FM algorithm and while using the RFA algorithm. however, the transient behavior is quite different. Noticeably, the FM algorithm encourages all of the transmitted powers to rise to the maximum power at the beginning of the simulation. This is an effect of the FM algorithm only considering the most recent SINR measurement when performing an update; at the beginning, all SINRs are below the target values, so they are increased drastically. It then takes a considerable amount of time (200 time steps) for the values to eventually stabilize, and it takes another 400 time steps for all of the powers to simultaneously decrease to eventually settle on the optimal powers.

Meanwhile, the RFA algorithm encourages a more gradual approach. Because of the role of averaging, when a transmitter receives a SINR measurement that is far below the target SINR, it slowly increases its transmitted power. With all transmitters doing this in tandem, this causes the trajectories 


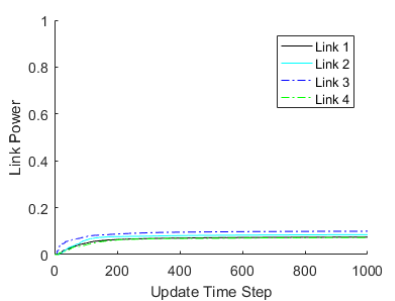

(a) Robust Feedback Averaging

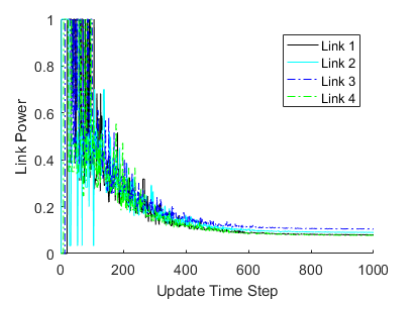

(b) Foschini-Miljanic
Fig. 2: Results of Experiment 2: Power marginal convergence behavior of the RFA algorithm (a) and FM algorithm (b) with a uniform random delay of $[0,100]$ time steps. Due to the out-of-order nature of the SINR measurements, the FM algorithm frequently unnecessarily increases the transmission power. Both algorithms appear to converge.

of the transmitted powers to approach the optimal power vector from below, rather than from above. This is significant, as the area under each curve represents the total power used by each transmitter. As seen in Figure 1, there is about an order of magnitude difference in the area under the curves in Figure 1(a) and Figure 1(b), which represents an order of magnitude power savings caused by RFA.

Experiment 2: The advantage of RFA is even more apparent when we allow the delays to be random. In our second simulation, we sample each delay $D_{i}^{t}$ individually from a uniform distribution over the integers, i.e. $D_{i}^{t} \sim$ $U(0,100)$. This is a more challenging environment than in the previous experiment, because the delay in the previous experiment, though it varied across channels, was constant with respect to a single channel. That meant that (after $t=$ 100) every transmitter received a single SINR measurement. In this experiment, we examine the impact of multiple SINR measurements per time step, and out-of-order SINR measurements.

Figure 2 shows the transmission power of each transmitter vs time. In Figure 2(a), we see a much more noisy convergence curve for the FM algorithm, with frequent spikes in the transmitted powers. Each spike in the plot after the initial transient behavior is due to an out-of-order SINR measurement; here, again, the "memoryless" property of the FM algorithm hinders its performance. The initial spike in power, which is similar to the behavior observed in Experiment 1, is again an unnecessary waste of power.

The RFA algorithm, on the other hand, does not cause any of the transmitters to send spikes in power when an out-of-order SINR is received. Similarly to Experiment 1, it converges from below to the optimal power vector in an energy-efficient manner.

\section{Stochastic and Time-Varying Channel}

We now turn to the case of a stochastic, time-varying channel with delay. We define a stochastic channel as one in which the gain matrices and noise evolve stochastically over time: $\left\{\mathbf{G}^{t}\right\}_{t=0}^{\infty},\left\{\eta^{t}\right\}_{t=0}^{\infty}$. For these experiments, we consider a stochastic channel that is drawn i.i.d. from a set of 2 deterministic channels, each with equal probability:

$$
\begin{aligned}
& \left(\mathbf{G}^{t}, \eta^{t}\right)=\left\{\begin{array}{lll}
\left(\mathbf{G}_{\mathbf{1}}, \eta_{1}\right), & \text { w.p. } & 0.5 \\
\left(\mathbf{G}_{\mathbf{2}}, \eta_{2}\right), & \text { w.p. } & 0.5,
\end{array}\right. \\
& \mathbf{G}_{\mathbf{1}}=\left[\begin{array}{ll}
2 & 5 \\
6 & 3
\end{array}\right] \eta_{1}=\left[\begin{array}{l}
0.1 \\
0.2
\end{array}\right] \\
& \mathbf{G}_{2}=\left[\begin{array}{ll}
5 & 3 \\
3 & 3
\end{array}\right] \eta_{2}=\left[\begin{array}{l}
0.15 \\
0.05
\end{array}\right]
\end{aligned}
$$

Again, we chose these matrices to present a challenging environment for the algorithms; even though there are only two channels, the $\mathbf{G}$ matrices dictate that the signal in each link will heavily interfere with the signals from the other link.

Experiment 3: First, we set $r_{i}^{*}=0.5$ and $p_{\max }=1.0$ as before. Using this $r_{i}^{*}$, we check channel feasibility by constructing $\mathbf{W}_{1}, \gamma_{1}, \mathbf{W}_{2}$, and $\gamma_{2}$. We see that $\lambda_{\max }\left(\mathbf{W}_{1}\right)=$ 1.34 and $\lambda_{\max }\left(\mathbf{W}_{2}\right)=0.46$. This indicates that the channel $G_{2}, \eta_{2}$ is feasible (by Definition 2, but the channel $G_{1}, \eta_{1}$ is infeasible. This means that, half of the time, the algorithms will be operating on an infeasible channel; the FM algorithm will not converge to the optimal power vector in this scenario.

However, the average channel,

$$
\overline{\mathbf{G}}=\left[\begin{array}{ll}
3.5 & 4 \\
4.5 & 3
\end{array}\right] \bar{\eta}=\left[\begin{array}{l}
0.125 \\
0.125
\end{array}\right],
$$

is feasible (with $\lambda_{\max }(\overline{\mathbf{W}})=0.79$ ). So, by the results shown in [12], the RFA algorithm allows all the link powers in this channel to converge to their optimal value (with no delay).

Now, we add delay to this stochastic channel. We again add uniform independent delay for each channel for each time step, i.e. $D_{i}^{t} \sim U(0,100)$. Figure 3 shows the results of this simulation, with Figures 3(a) and 3(b) illustrating the performance of RFA and FM, respectively.

We see that, again, the RFA algorithm converges smoothly to the optimal power vector from below, just as in previous experiments. However, the FM algorithm, as expected, does not converge. Since FM only relies on the most recently returned SINR measurement, whenever the channel is infeasible (which occurs in this experiment with probability 0.5 ), the transmitted powers will diverge. Of course, when the channel is feasible, the power vector decreases, so the power will only reach the maximum value if a long sequence of SINR measurements from the infeasible channel were returned sequentially, which will only happen with very low probability.

Experiment 4: For this experiment, we did not examine the performance of FM, but instead focused on the performance of RFA. We use the same stochastic channel as in Experiment 3, but here we vary $r_{i}^{*}$ between 0.5 and 0.9 (where we always set $r_{1}^{*}=r_{2}^{*}$ ).

As in Experiment 3, setting $r_{i}^{*}=0.5$ causes the average channel, $\overline{\mathbf{G}}=\left[\begin{array}{ll}3.5 & 4 \\ 4.5 & 3\end{array}\right] \bar{\eta}=\left[\begin{array}{l}0.125 \\ 0.125\end{array}\right]$ to be feasible (with $\left.\lambda_{\max }(\overline{\mathbf{W}})=0.79\right)$. However, when $r_{i}^{*}=0.9$, we reconstruct $\mathbf{W}_{i}$ and $\gamma_{i}$ and see that $\lambda_{\max }(\overline{\mathbf{W}})=1.31$ in this case. 


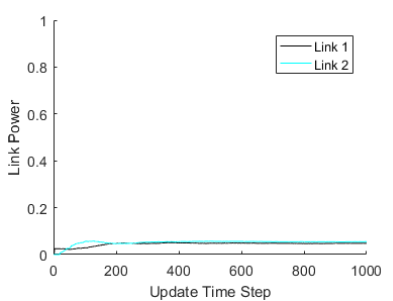

(a) Robust Feedback Averaging

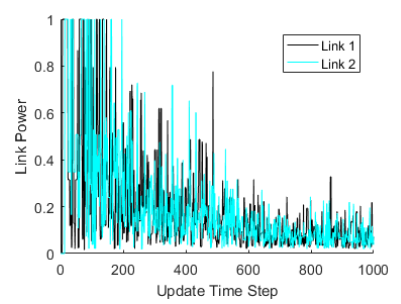

(b) Foschini-Miljanic
Fig. 3: Results of Experiment 3: power marginal convergence behavior in the presence of a stochastic, timevarying channel and uniformly random delay. The delays are sampled from uniform distribution: $D_{i}^{t} \sim U(0,100)$. Again, RFA (a) converges to the optimal average power, but the FM algorithm (b) does not converge, since the channel is infeasible half of the time.

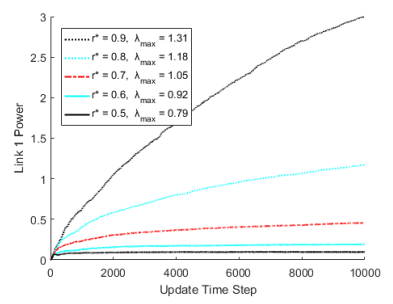

(a) Link 1 transmitted power

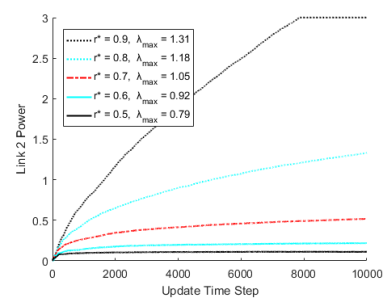

(b) Link 2 transmitted power
Fig. 4: Results of Experiment 4: power marginal convergence behavior of the RFA algorithm in the presence of a stochastic, time-varying channel and uniformly random delay $\left(D_{i}^{t} \sim U(0,100)\right)$. Five different values of $r_{i}^{*}$ are plotted here, and the corresponding $\lambda_{\max }(\overline{\mathbf{W}})$ for each is shown. The two links in the channel are plotted separately, with the transmitted power of Link 1 (a) above, and the transmitted power of Link 2 (b) below.

Therefore, the channel is on average infeasible, and we do not expect the link powers to converge when using RFA.

Figure 4 shows the results of this experiment. Unlike in the other figures, here Figure 4(a) shows the power transmitted by Link 1, and Figure 4(b) shows the power transmitted by Link 2. Each individual trace represents a different target SINR $r_{i}^{*}$. As we expect, when the convergence condition in Definition 2 holds, then the power vector converges to the optimal $\mathbf{p}^{*}$. But, when $\lambda_{\max }(\overline{\mathbf{W}})>1$, the powers diverge, and the powers diverge faster with larger $\lambda_{\max }(\overline{\mathbf{W}})$.

\section{REFERENCES}

[1] A. Goldsmith, Wireless communications. Cambridge university press, 2005.

[2] T. S. Rappaport et al., Wireless communications: principles and practice. prentice hall PTR New Jersey, 1996, vol. 2.

[3] G. J. Foschini and Z. Miljanic, "A simple distributed autonomous power control algorithm and its convergence," IEEE transactions on vehicular Technology, vol. 42, no. 4, pp. 641-646, 1993.

[4] Z. Zhou, D. Miller, N. Bambos, and P. Glynn, "A stochastic stability characterization of the foschini-miljanic algorithm in random wireless networks," in Global Communications Conference (GLOBECOM), 2016 IEEE. IEEE, 2016, pp. 1-6.
[5] R. D. Yates, "A framework for uplink power control in cellular radio systems," IEEE Journal on selected areas in communications, vol. 13, no. 7, pp. 1341-1347, 1995.

[6] Z. Zhou, P. Glynn, and N. Bambos, "Repeated games for power control in wireless communications: Equilibrium and regret," in Decision and Control (CDC), 2016 IEEE 55th Conference on. IEEE, 2016, pp. 3603-3610.

[7] S. Ulukus and R. D. Yates, "Stochastic power control for cellular radio systems," IEEE transactions on Communications, vol. 46, no. 6, pp. 784-798, 1998.

[8] Z. Han, Game theory in wireless and communication networks: theory, models, and applications. Cambridge University Press, 2012.

[9] I. Menache and A. Ozdaglar, "Network games: Theory, models, and dynamics," Synthesis Lectures on Communication Networks, vol. 4, no. 1, pp. 1-159, 2011.

[10] Z. Zhou and N. Bambos, "Wireless communications games in fixed and random environments," in Decision and Control (CDC), 2015 IEEE 54th Annual Conference on. IEEE, 2015, pp. 1637-1642.

[11] Z. Zhou, N. Bambos, and P. Glynn, "Deterministic and stochastic wireless networks games: Equilibrium, dynamics and price of anarchy," Operations Research, 2018.

[12] Z. Zhou, P. Mertikopoulos, A. Moustakas, S. Mehdian, N. Bambos, and P. Glynn, "Stable power control in wireless networks via dual averaging," in 2017 IEEE Global Communications Conference (GLOBECOM), Dec 2017, pp. 1-6.

[13] T. Charalambous, I. Lestas, and G. Vinnicombe, "On the stability of the foschini-miljanic algorithm with time-delays," in Decision and Control, 2008. CDC 2008. 47th IEEE Conference on. IEEE, 2008, pp. 2991-2996.

[14] T. Charalambous and Y. Ariba, "On the stability of a power control algorithm for wireless networks in the presence of time-varying delays," in Control Conference (ECC), 2009 European. IEEE, 2009, pp. 2936-2941.

[15] D. U. Campos-Delgado and M. Luna-Rivera, "Characterization of stability margins of the foschini-miljanic power allocation strategy under constant and time-varying delays," in Electrical Engineering Computing Science and Automatic Control (CCE), 2011 8th International Conference on. IEEE, 2011, pp. 1-6.

[16] D. Mitra, "An asynchronous distributed algorithm for power control in cellular radio systems," KLUWER INTERNATIONAL SERIES IN ENGINEERING AND COMPUTER SCIENCE, pp. 177-177, 1994.

[17] Y. Wei, J. Qiu, P. Shi, and M. Chadli, "Fixed-order piecewise-affine output feedback controller for fuzzy-affine-model-based nonlinear systems with time-varying delay," IEEE Transactions on Circuits and Systems I: Regular Papers, vol. 64, no. 4, pp. 945-958, 2017.

[18] J. Qiu, T. Wang, S. Yin, and H. Gao, "Data-based optimal control for networked double-layer industrial processes," IEEE Transactions on Industrial Electronics, vol. 64, no. 5, pp. 4179-4186, 2017.

[19] Z. Han, D. Niyato, W. Saad, T. Başar, and A. Hjørungnes, Game Theory in Wireless and Communication Networks: Theory, Models, and Applications. Cambridge University Press, 2011. [Online] Available: https://books.google.com/books?id=mvaUAwAAQBAJ

[20] P. C. Weeraddana, M. Codreanu, M. Latva-aho, A. Ephremides, C. Fischione et al., "Weighted sum-rate maximization in wireless networks: A review," Foundations and Trends $®$ in Networking, vol. 6 , no. 1-2, pp. 1-163, 2012.

[21] C. W. Tan, "Wireless network optimization by perron-frobenius theory," in Information Sciences and Systems (CISS), 2014 48th Annual Conference on. IEEE, 2014, pp. 1-6.

[22] Y. Nesterov, "Primal-dual subgradient methods for convex problems,' Mathematical programming, vol. 120, no. 1, pp. 221-259, 2009.

[23] Z. Zhou, P. Mertikopoulos, N. Bambos, S. Boyd, and P. W. Glynn, "Stochastic mirror descent in variationally coherent optimization problems," in Advances in Neural Information Processing Systems, 2017, pp. 7040-7049.

[24] P. Mertikopoulos and Z. Zhou, "Learning in games with continuous action sets and unknown payoff functions," Mathematical Programming, Mar 2018. [Online]. Available: https://doi.org/10.1007/s10107-018-1254-8 